\title{
Investigation of the $\mathrm{Si} / \mathrm{TiO}_{2} /$ Electrolyte Interface using Operando Tender X-ray Photoelectron Spectroscopy
}

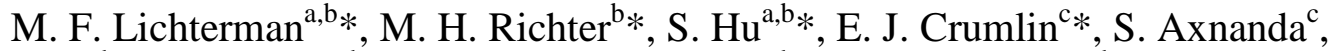

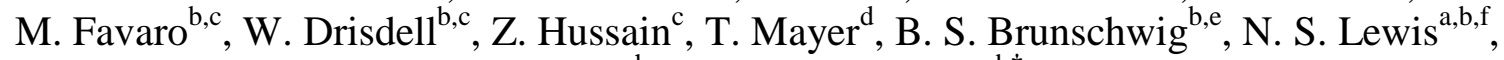 \\ Z. Liu ${ }^{\mathrm{c}, \mathrm{g}, \mathrm{h}}$, and H.-J. Lewerenz ${ }^{\mathrm{b} \ddagger}$ \\ ${ }^{a}$ Division of Chemistry and Chemical Engineering, California Institute of Technology, \\ Pasadena, CA 91125, USA. \\ b Joint Center for Artificial Photosynthesis, California Institute of Technology, Pasadena, \\ CA 91125, USA. \\ ${ }^{\mathrm{c}}$ Advanced Light Source, Lawrence Berkeley National Laboratory, Berkeley, CA 94720 \\ ${ }^{\mathrm{d}}$ Surface Science Division, Materials Science Department, Darmstadt University of \\ Technology, 64287 Darmstadt, Germany. \\ e Beckman Institute, California Institute of Technology, Pasadena, CA 91125, USA \\ ${ }_{\mathrm{f}}^{\mathrm{f}}$ Kavli Nanoscience Institute, California Institute of Technology, Pasadena, CA 91125, \\ USA. \\ ${ }^{\mathrm{g}}$ State Key Laboratory of Functional Materials for Informatics, Shanghai Institute of \\ Microsystem and Information Technology, Chinese Academy of Sciences, Shanghai \\ 200050, China. \\ ${ }^{\mathrm{h}}$ School of Physical Science and Technology, ShanghaiTech University, Shanghai \\ 200031, China. \\ ‡Corresponding author E-mail: lewerenz@caltech.edu
}

\begin{abstract}
Semiconductor-electrolyte interfaces allow for the creation of photoactive semiconductor systems that have band bending and other characteristics analogous to semiconductor-metal junctions (Schottky junctions). We demonstrate herein that XPS measurements can be obtained on a full three-electrode electrochemical system under potentiostatic control by use of tender X-rays to provide photoelectrons with sufficient kinetic energy to penetrate through a thin electrolyte overlayer on a portion of the working electrode. The response of the photoelectron binding energies to variations in applied voltage demonstrates that the XPS investigation works in an operando manner to elucidate the energetics of such interfaces.
\end{abstract}

\section{Introduction}

Photoelectrochemical cells have garnered much attention for potential applications in solar-energy conversion and storage (1). Numerous experimental techniques have been deployed to further the understanding of the semiconductor/liquid junctions that are at the heart of such systems (2-11). Semiconductor-metal (Schottky) junctions have been thoroughly analyzed by surface analyses and optoelectronic methods, whereas the energetic relations at semiconductor/liquid junctions remain elusive. The inherent difficulty results from the requirement that a conductive electrolyte must be present on 
the surface of the semiconductor, precluding the use of conventional surface-science methods to probe the semiconductor/liquid interface of an operating photoelectrode.

Ambient-Pressure X-ray Photoelectron Spectroscopy (AP-XPS) has been used to investigate gas-solid and gas-liquid interfaces as well as related processes such as oxygen reduction on fuel cell cathodes (12), mechanisms of heterogeneous catalysis (13) and formation of metal oxide overlayers (14). Herein, we describe the use of AP-XPS, with real-time control of the electrochemical potential, through a conductive aqueous electrolyte on a semiconductor/liquid junction. A $\sim 13 \mathrm{~nm}$ thick hanging meniscus on the (semiconductor) working electrode in a three-electrode setup permitted electrochemical control of the solid/liquid interface. Differential pumping allowed maintaining the XPS analysis chamber at $\sim 15$ Torr while the analyzer was kept at a much lower pressure (15).

$\mathrm{TiO}_{2}$ has recently been reported to provide a protective layer that permits the use of small band-gap semiconductors (such as $\mathrm{Si}$, GaAs, CdTe, etc.) as photoanodes with extended stability relative to unprotected surfaces of such semiconductors. In this application, the photogenerated holes transport through the $\mathrm{TiO}_{2}$ during the photoelectrochemical oxidation of $\mathrm{H}_{2} \mathrm{O}$ to $\mathrm{O}_{2}(16,17)$. Herein we describe the use of APXPS to further characterize these $\mathrm{TiO}_{2}$ /electrolyte and $\mathrm{TiO}_{2} / \mathrm{Ni} /$ electrolyte interfaces.

\section{Experimental}

Films of $\mathrm{TiO}_{2}$ deposited by atomic-layer deposition $(16,17)$, were prepared on degenerately doped p-type silicon ("p ${ }^{+}-\mathrm{Si}$ ") substrates. Si (100) wafers with a resistivity, $\rho<.005 \Omega * \mathrm{~cm}$, were first cleaned with an oxidizing etch by soaking in a $3: 1(\mathrm{v}: \mathrm{v})$ "piranha" solution of concentrated $\mathrm{H}_{2} \mathrm{SO}_{4}(98 \%)$ to $30 \% \mathrm{H}_{2} \mathrm{O}_{2}$ for 2 min, and then etching for $10 \mathrm{~s}$ in a $10 \%$ (by volume) solution of $\mathrm{HF}(\mathrm{aq})$. The wafers were then etched in a 5:1:1 (by volume) solution of $\mathrm{H}_{2} \mathrm{O}, 36 \%$ hydrochloric acid, and $30 \%$ hydrogen peroxide for $10 \mathrm{~min}$ at $75{ }^{\circ} \mathrm{C}$. The $\mathrm{TiO}_{2}$ was deposited from a tetrakis(dimethylamido)titanium (TDMAT) precursor. A $0.1 \mathrm{~s}$ pulse of TDMAT was followed by $15 \mathrm{~s}$ purge of $\mathrm{N}_{2}$ at 20 sccm, following by a $0.015 \mathrm{~s}$ pulse of $\mathrm{H}_{2} \mathrm{O}$ before another $15 \mathrm{~s}$ purge with $\mathrm{N}_{2}$. This process was repeated for 1500 cycles to provide films $\sim 70 \mathrm{~nm}$ in thickness. Where desired, Ni was deposited at a RF sputtering power of $150 \mathrm{~W}$ for $80 \mathrm{~s}$. Atomic-force microscopy (AFM) images attested to the smoothness of these films on the nanometer scale.

To prepare electrodes for operando AP-XPS, strips of the $\mathrm{p}^{+}-\mathrm{Si} / \mathrm{TiO}_{2} /(\mathrm{Ni})$ wafers were cut into $1 \mathrm{~cm} \times 3.5 \mathrm{~cm}$ rectangles. Highly doped p-type silicon $\left(\mathrm{p}^{+}\right)$was used simultaneously as a support material as well as to provide an effective back contact to the ALD- $\mathrm{TiO}_{2}$ (Figure 1). The ohmic contact at the back of the semiconductor was connected to the photoelectron analyzer to provide a high conductivity ground for the sample. To make ohmic contact, an In/Ga eutectic was scribed into the back of the Si wafer, and Ag paint was then used to contact the electrode to a strip of $\mathrm{Cu}$ tape that was supported on a $0.8 \mathrm{~cm}$ x $3 \mathrm{~cm}$ glass slide. 


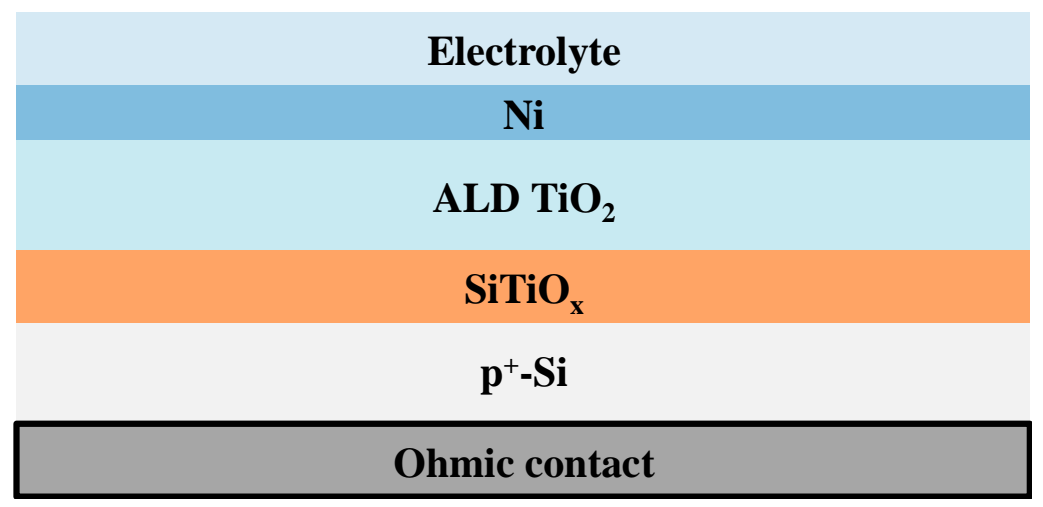

Figure 1. Schematic of the layer stack investigated by operando XPS. The thicknesses of the various layers are not drawn to scale (see text). On bare electrodes, no nickel was present between the ALD $\mathrm{TiO}_{2}$ and the electrolyte.

To perform the operando AP-XPS measurements, the $\mathrm{p}^{+}-\mathrm{Si} / \mathrm{TiO}_{2} /(\mathrm{Ni})$ electrode was mounted and contacted by a three-axis manipulator onto which the $\mathrm{Ag} / \mathrm{AgCl}$ reference electrode (eDAQ) and a platinum foil counter electrode were also mounted and separately contacted. To collect data, the sample was "dipped" into the electrolyte before a potential was set using the potentiostat. The electrode was then raised to location that produced a thin film of the electrolyte $(\sim 13 \mathrm{~nm})$ at the XPS sampling location, as determined by IMFP calculations based on the intensity of the signal observed on the detector relative to the X-ray intensity incident onto the system.

AP-XPS data were collected on a Scienta R4000 HiPP-2 system in which the photoelectron collection cone was aligned to the beamline X-Ray spot at a distance of $\sim 300 \mu \mathrm{m}$. Beamline 9.3.1 at the Advanced Light Source was used to provide Tender $\mathrm{X}$-Rays of $4 \mathrm{keV}$ photon energy out of a possible photon energy range of 2.3-5.2 keV, with a resolving power of $\mathrm{E} / \Delta \mathrm{E}=3000-7200$. Photoelectrons for the Ti $2 \mathrm{p}$ level were collected in kinetic energy ranges of $3538 \pm 8 \mathrm{eV}$. Multiple scans [10 - 15] were required to achieve a satisfactory signal-to-noise ratio.

\section{Results and Discussion}

Mott-Schottky measurements of the inverse square of the differential capacitance vs potential showed that the $\mathrm{p}^{+}-\mathrm{Si} / \mathrm{TiO}_{2}$ system adopted a flat-band position at a potential of $\sim-0.7 \mathrm{~V}$ vs the reversible hydrogen electrode, RHE, in $1.0 \mathrm{M} \mathrm{KOH}(\mathrm{aq})$ (Figure 2). Other measurements have indicated that an ohmic contact was formed between $\mathrm{p}^{+}-\mathrm{Si}$ and the ALD- $\mathrm{TiO}_{2}$ used here (16). As will be demonstrated below, a stack consisting of $\mathrm{p}^{+}$$\mathrm{Si} / \mathrm{TiO}_{2} / \mathrm{Ni}$, when cycled electrochemically in a fast redox couple solution, displayed ohmic behavior, indicating effective ohmic metallurgical contacts throughout the structure.

The measured XPS data and the related energy-band alignments can be influenced by surface roughness effects, particularly if the semiconductor is highly doped. The electrolyte makes a conformal contact to even a very rough semiconductor surface, so a larger volume fraction of the semiconductor surface can contain a space-charge region on a roughened semiconductor surface than on a flat surface. For a semiconductor with a space-charge region width on the order of nanometers, this behavior may influence the position and width of an XPS peak. Moreover, the band energetics directly at the surface 
will be overestimated in an XPS spectrum for semiconductors with surface roughness on the same scale as the depth of the space-charge region, relative to the situation for a flat surface. Specifically, in situations where surface roughness allows a much larger fraction of the semiconductor volume to be described as in the space-charge region, the effect can be large, because of the binding energy change $\Delta E_{B}(\mathrm{x})$ near the surface, where $\mathrm{x} \sim 0$ (equation 1), will dominate:

$$
\left.\Delta E_{B}(x)\right|_{0} ^{d}=-q\left[U_{0}-\frac{2 \cdot U_{0}}{d^{2}}\left(d \cdot x-\frac{x^{2}}{2}\right)\right]
$$

Here $U_{0}$ is the applied potential to the back of the semiconductor, $d$ is the thickness of the semiconductor space-charge region, and $x$ is the distance to the surface.

However, for surface roughness on a length scale that is substantially smaller than the extension of the semiconductor space-charge region, or with a space-charge region that is considerably larger than the penetration depth of the technique, this effect is essentially negligible.
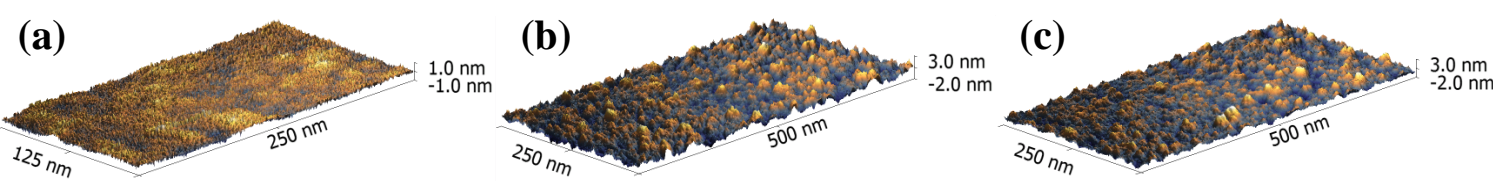

Figure 2. 3D AFM images of (a) bare silicon, (b) $\sim 70 \mathrm{~nm}$ thick $\mathrm{TiO}_{2}$ on silicon, and (c) the same as (b) but with $\sim 2 \mathrm{~nm} \mathrm{Ni}$.

The $\mathrm{TiO}_{2}$ used in this study had a space-charge region on the order of tens of nanometers at $1 \mathrm{~V}$ of applied bias, as calculated from the dopant densities determined by the MottSchottky analysis. Thus, sample roughness effects on the collected data should be minimal in the absence of a macroscopically rough surface. As shown in Figure 2, the roughness of the $\mathrm{TiO}_{2}$ samples is on the order of $\sim 1 \mathrm{~nm}$ across tens of nanometers, hence surface topography should not significantly influence the results observed herein by APXPS. 
(a)

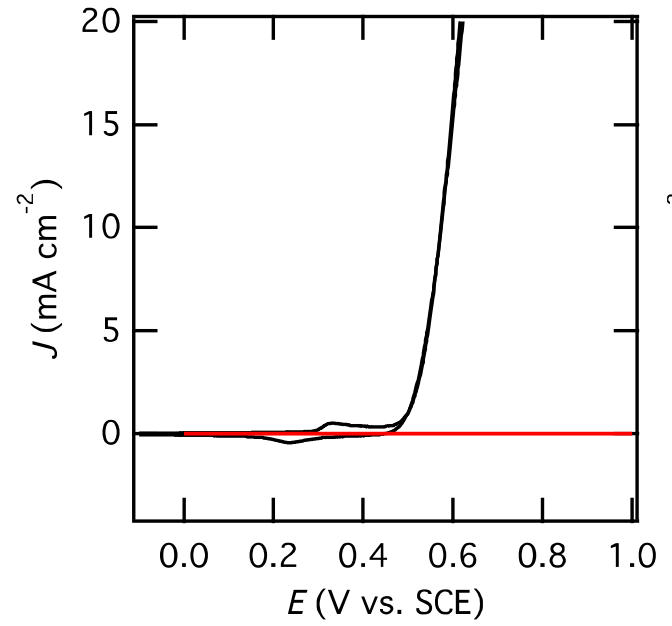

(b)

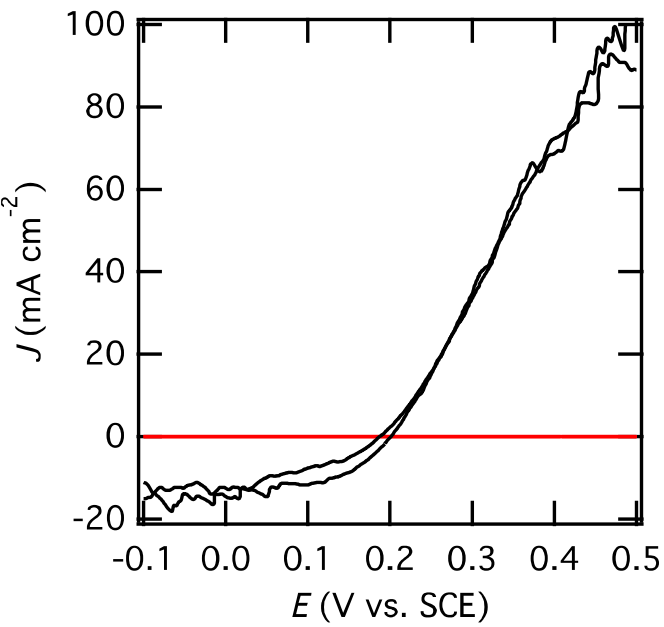

Figure 3. Dark $J-E$ measurements for $\mathrm{p}^{+}-\mathrm{Si} / \mathrm{TiO}_{2}$ (red) and $\mathrm{p}^{+}-\mathrm{Si} / \mathrm{TiO}_{2} / \mathrm{Ni}$ (black) electrodes in (a) $1 \mathrm{M} \mathrm{KOH}$ and (b) a solution containing $50 \mathrm{mM}$ potassium ferricyanide and $350 \mathrm{mM}$ potassium ferrocyanide. The noisy cathodic and anodic currents at $\sim-20$ $\mathrm{mA} \cdot \mathrm{cm}^{-2}$ and $100 \mathrm{~mA} \cdot \mathrm{cm}^{-2}$ are limited by ferri/ferrocyanide concentration and transport.

Electrochemical experiments were undertaken to examine the nature of the $\mathrm{TiO}_{2} /$ electrolyte and $\mathrm{TiO}_{2} / \mathrm{Ni} /$ electrolyte junction before operando measurements were performed. As demonstrated in Figure 3, electrodes consisting of $\mathrm{p}^{+}-\mathrm{Si} / \mathrm{TiO}_{2}$ in an aqueous ferri/ferrocyanide solution showed anodically rectifying behavior for the kinetically slow process of water oxidation as well as for the kinetically rapid process of ferrocyanide oxidation. However, with $\mathrm{Ni}$ present on the electrode surface, both processes exhibited little charge-transfer resistance; while the enhanced water oxidation current density might be argued to originate from enhanced catalysis facilitated by the presence of $\mathrm{Ni}$, the change in the observed electrochemical behavior in contact with the ferri/ferrocyanide redox system strongly indicates that the addition of Ni drastically improved the ohmic conductivity of $\mathrm{TiO}_{2} /$ electrolyte interface and allowed for efficient hole-charge transport. 
(a)

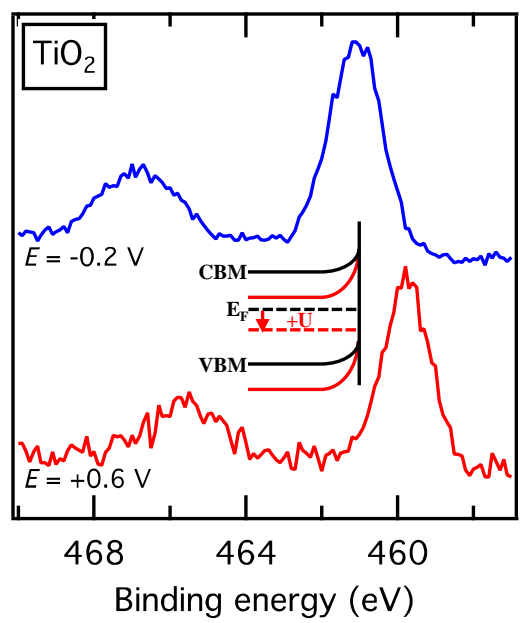

(b)

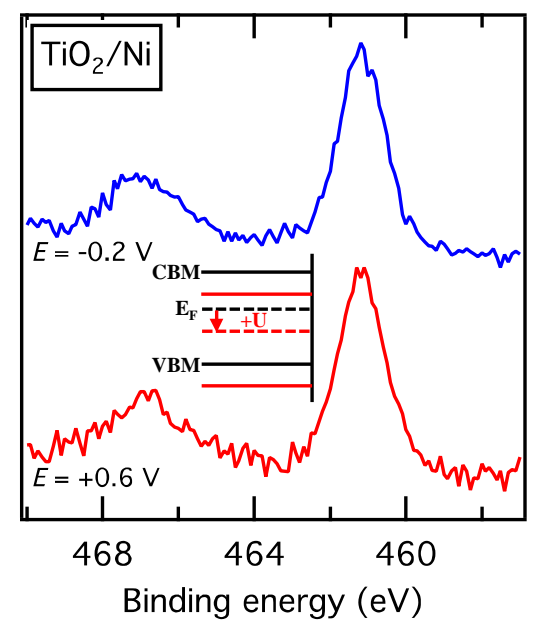

Figure 4. (a) $\mathrm{Ti} 2 \mathrm{p}$ XPS spectra of the bare $\mathrm{TiO}_{2}$ electrode in $1.0 \mathrm{M} \mathrm{KOH}(\mathrm{aq})$ at $-0.2 \mathrm{~V}$ and $+0.6 \mathrm{~V}$ vs $\mathrm{Ag} / \mathrm{AgCl}$. The inset shows the change in band bending as the potential was made more positive. (b) $\mathrm{Ti} 2 \mathrm{p}$ XPS spectra of a $\mathrm{TiO}_{2} / \mathrm{Ni}$ electrode under the same conditions. The inset shows the manner in which the $\mathrm{TiO}_{2}$ band bending was invariant with respect to the applied potential.

As shown in Figure 4a, for a bare $\mathrm{TiO}_{2}$ surface, the observed binding energy shifted in accord with a change in the applied potential. Thus, this system followed the ideal semiconductor/liquid junction behavior in this potential range, as described in the inset. Specifically, as the Fermi level of the semiconductor shifted to a more positive potential, the fixed positions of the band edges in the semiconductor/liquid junction caused the observed binding energies to decrease. In contrast, the metallized $\mathrm{TiO}_{2}$ film (Figure $4 \mathrm{~b}$ ) exhibited very different behavior. Specifically, effectively little or no shift was observed in the $\mathrm{TiO}_{2}$ core-level emission peak positions as the applied potential was varied. This behavior is consistent with the observation that metallization (using $\mathrm{Ni}$ ) of the $\mathrm{TiO}_{2}$ surface allows for charge-carrier transport (16). These results therefore demonstrate that the $\mathrm{Ni}$ on the surface pins the semiconductor energetics to that of the metal instead of the electrolyte, and plays a key role in allowing for charge conduction to take place. Effectively, metallization allows the semiconductor band edges to shift with potential across a wide potential range.

\section{Conclusion}

Operando XPS technique can be used to determine the nature of band energetics in a semiconductor-liquid junction. The nature of the junction formed can be tuned by appropriate modification of the semiconductor surface, which has crucial effects on the nature of space-charge region formation or charge conduction therein. Further experiments are underway to elaborate in more detail the diverse influences of semiconductor doping, electrolyte choice, and other relevant variables on the system. 


\section{Acknowledgments}

This work was supported through the Office of Science of the U.S. Department of Energy (DOE) under award no. DE-SC0004993 to the Joint Center for Artificial Photosynthesis, a DOE Energy Innovation Hub. The Advanced Light Source is supported by the Director, Office of Science, Office of Basic Energy Sciences, of the U.S. Department of Energy under Contract No. DE-AC02-05CH11231. We thank Dr. Philip Ross for contributions to the conceptual development of the AP-XPS end-station and experimental design.

\section{References}

1. H. Gerischer, J. Electroanal. Chem. and Interfacial Electrochem., 58, 263 (1975).

2. A. J. Bard, J. Phys. Chem., 86, 172 (1982).

3. A. J. Bard, A. B. Bocarsly, F. R. F. Fan, E. G. Walton, and M. S. Wrighton, J. Am. Chem. Soc., 102, 3671 (1980).

4. A. Heller, K. C. Chang, and B. Miller, J. Electrochem. Soc., 124, 697 (1977).

5. A. Heller, B. Miller, and F. A. Thiel, Appl. Phys. Lett., 38, 282 (1981).

6. N. S. Lewis, J. Electrochem. Soc., 131, 2496 (1984).

7. B. Miller, A. Heller, S. Menezes, and H. J. Lewerenz, Faraday Discuss., 70, 223 (1980).

8. H. J. Lewerenz, Chem. Soc. Rev., 26, 239 (1997).

9. K. Jacobi, M. Gruyters, P. Geng, T. Bitzer, M. Aggour, S. Rauscher, and H. J. Lewerenz, Physical Review B, 51, 5437 (1995).

10. H. J. Lewerenz, M. Aggour, C. Murrell, M. Kanis, H. Jungblut, J. Jakubowicz, P. A. Cox, S. A. Campbell, P. Hoffmann, and D. Schmeißer, J. Electrochem. Soc., 150, E185 (2003).

11. M. Letilly, K. Skorupska, and H.-J. Lewerenz, J. Phys. Chem. C, 117, 16381 (2013).

12. Y.-C. Lu, E. J. Crumlin, G. M. Veith, J. R. Harding, E. Mutoro, L. Baggetto, N. J. Dudney, Z. Liu, and Y. Shao-Horn, Sci. Rep., 2, 715 (2012).

13. V. V. Kaichev, I. P. Prosvirin, and V. I. Bukhtiyarov, J Struct Chem, 52, 90 (2011).

14. A. Y. Klyushin, T. C. R. Rocha, M. Havecker, A. Knop-Gericke, and R. Schlögl, Phys. Chem. Chem. Phys., 16, 7881 (2014).

15. S. Axnanda, E. J. Crumlin, B. Mao, S. Rani, R. Chang, W. C. Stolte, P. G. Karlsson, M. O. M. Edwards, M. Lundqvist, R. Moberg, P. Ross, Z. Hussain, and Z. Liu, Submitted (2015).

16. S. Hu, M. R. Shaner, J. A. Beardslee, M. F. Lichterman, B. S. Brunschwig, and N. S. Lewis, Science, 344, 1005 (2014).

17. M. F. Lichterman, A. I. Carim, M. T. McDowell, S. Hu, H. B. Gray, B. S. Brunschwig, and N. S. Lewis, Energy Environ. Sci., 7, 3334 (2014). 